\section{Hypoxic gas mixture delivery due to malfunctioning inlet port of a Select-a-Tec vaporiser manifold}

To the Editor:

A potentially serious malfunction of an OHMEDA Modulus I gas machine occurred which allowed a hypoxic mixture to be delivered to a patient. At the time of the incident, the source of the problem was identified as a malfunctioning inlet valve of the Select-a-Tec SM vaporizer manifold. When a mounted Tec 4 vaporizer was set to a low concentration ( 0.25 per cent) the valve partially occluded the gas flow. This caused a back pressure which preferentially dropped the oxygen flow while maintaining the $\mathrm{N}_{2} \mathrm{O}$ flow, Valve replacement corrected the problem. No patient injury occurred.

Subsequent investigations by OHMEDA have revealed that this situation can occur when the two springs in the vaporizer manifold port valve are reversed (due to a servicing error) and the vaporizer setting is between 0 and 0.3 per cent. OHMEDA fecls that improved service standards should eliminate the problem. They state that this occurrence is rare and do not feel that design modifications are warranted. OHMEDA has devised a test (see Medical Device Safety Alert which follows) to detect the problem following servicing, at the time of the pre-use checkout. If performed as outlined, this test should satisfactorily uncover this problem. However, not all OHMEDA machines are serviced by OHMEDA technicians and also, not all anaesthetists perform routine preoperative checks.

Ohmeda's investigations have quantified my obscrvation that outflow resistance proximal to the pressure relief valve does allow a preferential flow of $\mathrm{N}_{2} \mathrm{O}$ over $\mathrm{O}_{2}$, that is, delivery of a hypoxic mixture. The higher $\mathrm{N}_{2} \mathrm{O}$ pressure from the secondary regulator is necessary for the functioning of the chain drive $\mathrm{N}_{2} \mathrm{O} / \mathrm{O}_{2}$ proportion limiting control. Without this pressure difference, the OHMEDA representative stated, there would be a mechanical problem with this linkage. It would seem, therefore, that to eliminate the risk of a hypoxic mixture from a partial obstruction by increasing the $\mathrm{O}_{2}$ pressure to equal or exceed that of $\mathrm{N}_{2} \mathrm{O}$ would entail a major re-design of the machine.

This incident demonstrates that despite apparent safeguards, a modern sophisticated anaesthesia machine can malfunction. The importance of vigilance and a functioning $\mathrm{O}_{2}$ analyzer were once again appreciated. The service policy changes and testing protocol outlined by $\mathrm{OH}-$ MEDA should help to reduce the occurrence of this particular problem, yet I cannot help wondering whether certain design features could be modified to further minimize hypoxic risk.

\section{John Riendl MD FRCPC \\ Department of Anaesthesia}

Dr. Evcrett Chalmers Hospital

Fredcricton, New Brunswick

E3B 5N5

Present address:

Department of Anaesthesia

Nanaimo Regional Hospital

Nanaimo, British Columbia

V9S 2B7

\section{EDITORIAL NOTE}

OHMEDA, Madison, Wisconsin, submitted the following Alert for publication in association with the above letter. The Alert has been reproduced without change.

\section{Urgent - Medical Device Safety Alert}

\section{May 1, 1987}

Ohmeda, Ohlo and Fraser Harlake (Boyle) Anesthesia Machines that are equipped with Selectatec Vaporizer Manifolds

Dear Chief Anesthesiologist:

Ohmeda has become aware of a condition that could result in the preferential reduction of total gas flow from the above listed anesthesia machines. This can occur only when using the SELECTATEC manifold in conjunction with a very low setting on the Tec 4 vaporizer. In extremely rare cases, this could cause the machine to deliver a hypoxic mixture. While only two complaints have been reported, neither of which resulted in patient injury, this is a potentially serious matter that deserves immediate attention, but that is readily solvable.

This problem occurs only with anesthesia machines that are equipped with the SELECTATEC manifolds (Figure) to which an Ohmeda Tec 4 vaporizer can be mounted. The cause was lound to be a high back pressure at very low output vaporizer levels, generated by improper functioning of the manifold port valve. This improper functioning could be the result of improper reassembly after servicing. The resultant back pressure will cause a reduction in the gas flow when the vaporizer is set to very low output levels (less than $\frac{1}{2}$ per cent).

Although we are aware of only two complaints, Onmeda wishes to minimize the possibility of any such additional events. Therefore, a test has been devised to detect if this reduction of flow can occur on your machine.

Ohmeda requests that you perform the following test on any machines that are equipped with the SELECTATEC manifold.

1 Set the oxygen flow rate 106 LPM.

2 Turn on the Tec 4 vaporizer slowly, advancing slowly "click by click" the vaporizer knob, through a percentage 